Revista Ingeniería y Región. 2015;14(2):99-112

\title{
Influencia de la presión de contacto sobre los parámetros de diseño de pavimentos asfálticos usando modelos de elementos finitos
}

\section{Influence of the contact pressure on the design parameters of asphalt pavements using finite element models}

\author{
Myriam R. Pallares Muñoz ${ }^{1}$, Julián A. Pulecio Díaz²
}

\begin{abstract}
Resumen
Se evalúa la influencia de la presión de contacto de una carga dual sobre los parámetros de diseño de pavimentos asfalticos por medio del modelador de elementos finitos EverStressFE 1.0 intentando reproducir de una manera más ajustada la forma de la huella y la intensidad de esfuerzo generados por las llantas de los vehículos. Para la validación se empleó el software multicapa elástico EverStress@ $(5.0$. Los resultados de las deformaciones llevaron a concluir que los diseños de pavimentos asfálticos realizados con métodos analíticos pueden estar ligeramente sobredimensionados y en consecuencia elevar los costos de construcción de los pavimentos. Este estudio traza una ruta para analizar la sensibilidad de distintos factores que pueden afectar el diseño de pavimentos asfalticos. Se espera en próximas investigaciones integrar condiciones dinámicas introduciendo resultados obtenidos de pruebas de campo a escala real.
\end{abstract}

Palabras clave: EverStressFE1.0; EverStress $(\mathbb{R} 5.0$; modelado tridimensional de pavimentos; área de contacto; huella de llanta; distribución de presión de inflado; modelado de pavimentos con software libre.

\begin{abstract}
The influence of a dual tire pressure on the design parameters of asphalt pavements using finite element modeling EverStressFEC1.0 is evaluated. This is trying to represent more adjusted the footprint shape and intensity of stress generated by the tires of vehicles. To validate the elastic multilayer EverStress $\mathbb{C 5 . 0}$ software was used. The results of the deformations can be concluded that the asphalt pavement designs made with analytical methods may be slightly oversized and consequently increase the cost of construction of pavements. This study marks a route to analyze the sensitivity of various factors that may affect the design of asphalt pavements. Future research is expected to integrate dynamic conditions by introducing results of field tests to full scale.
\end{abstract}

Key words: EverStressFEC1.0; EverStress $C 5.0$; three-dimensional modeling of pavements; contact area; tire tread; tire-footprint-pressure-distribution; free-software pavement modeling.

1 Magíster Métodos Numéricos en Ingeniería. Universidad Surcolombiana. Avenida Pastrana Borrero Carrera $1^{\text {a }}$, NeivaColombia, myriam.pallares@usco.edu.co

2 Magister Construcción Obras Viales. Universidad Surcolombiana. Avenida Pastrana Borrero Carrera 1, Neiva-Colombia, julpul7@hotmail.com 


\section{Introducción}

En la actualidad en el diseño de estructuras de pavimento se emplean métodos analíticos y/o modelos numéricos, definiendo para ello: el eje de diseño (eje simple de 13T de cuatro ruedas, 2 en cada extremo), el espesor de las capas y las características de los materiales. De manera que se pueda calcular las deformaciones a tensión en las fibras inferiores de las capas asfálticas y a compresión en la subrasante, provocadas por la presión de contacto de la carga de diseño que tradicionalmente se aplica de manera homogénea, en un área circular sobre la superficie de rodadura como se ilustra en la figura 1. Esta variable ha sido objeto de estudio de investigaciones que afirman que "la presión de contacto no se distribuye uniformemente, lo que proporciona alguna información para justificar por qué el supuesto de área de contacto convencional está incorrecto" (Xia, 2010). Esta premisa ha sentado las bases de un estudio para evaluar la influencia de la presión de contacto de una carga dual sobre los parámetros de diseño de pavimentos asfalticos (deformaciones horizontales " $E x x$ " y verticales " $\varepsilon z z$ "). Para este propósito se empleó el modelador tridimensional de elementos finitos EverStressFE 1.0 que permite reproducir de una manera más ajustada la forma de la huella y la intensidad de esfuerzo generados por las llantas de los vehículos de diseño (figuras 2 y 3) y el software multicapa elástico EverStress@ 5.0 para validar los resultados.

\section{Métodos}

El análisis se abordó por medio de los modeladores computacionales EverStress (C) 5.0 y EverStressFE1.0; el primero se utilizó para validar los resultados de los modelos de elementos finitos dada su versatilidad y funcionalidad. La estructura de pavimento asfáltico representada es semi-flexible compuesta por una base de grava (GB) y una capa de rodadura de concreto asfaltico (BBSG) como se muestra en la figura 4.

Las dimensiones de las capas constitutivas de la estructura de pavimento modelado se presentan en la Tabla 1. La condición de frontera considerada para la subrasante es semi-infinita y como es común en el dimensionamiento de este tipo de estructuras se trabajaron las interfaces ligadas.

Los módulos de elasticidad tomados como referencia para cada una de las capas de acuerdo a las condiciones de trabajo del pavimento se muestran en la Tabla 2. El módulo de elasticidad de la subrasante conocido

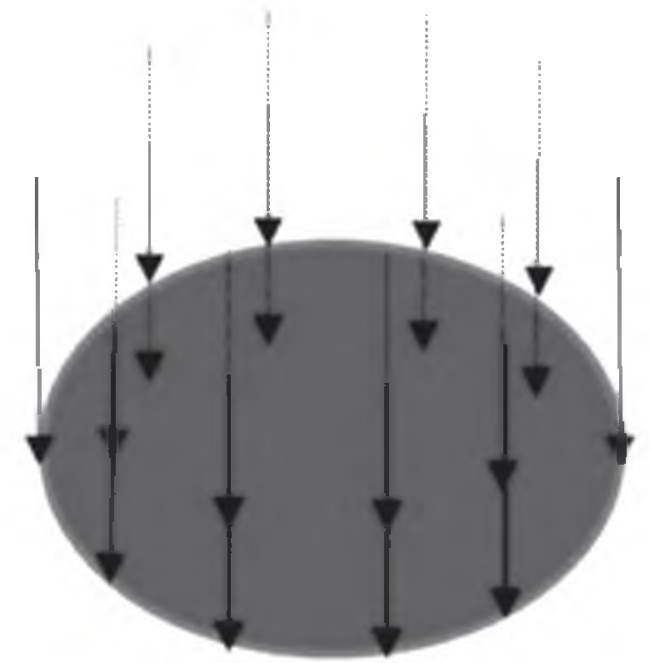

Figura 1. Área de contacto circular. Fuente: propia.
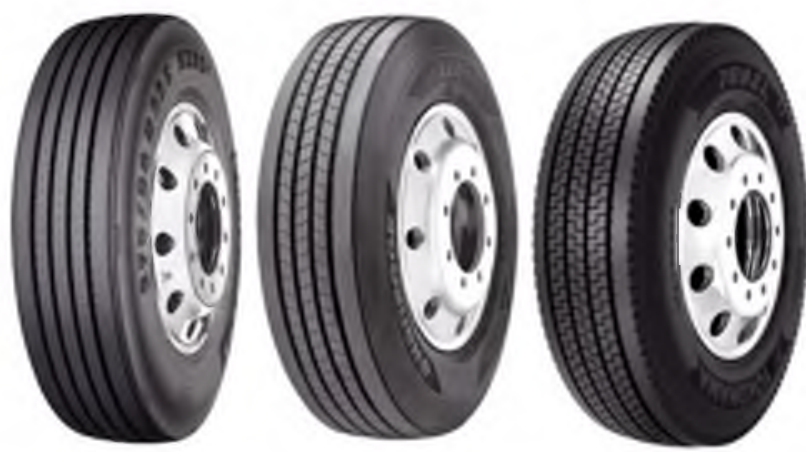

Figura 2. Formas típicas de llanta en el mercado automotor Fuente: propia.
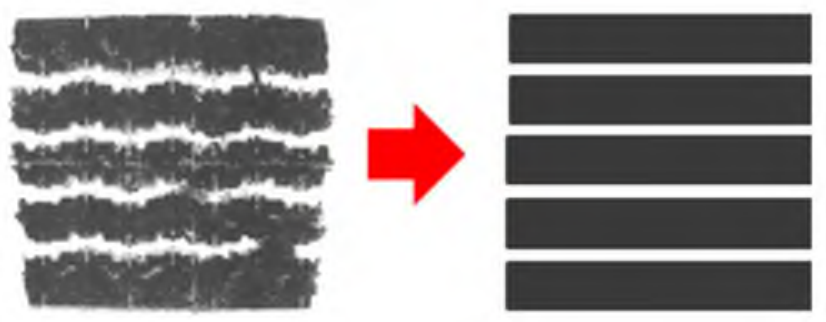

Figura 3. Huella rectangular. Fuente: propia

Tabla 1.Valores típicos de espesores de capa de una estructura de pavimento semi-flexible.

Capa

Espesor (m)

Capa de rodadura

(concreto asfaltico BBSG)

0.060

Base asfáltica (grava asfáltica GB)

0.180

Subrasante

semi-infinita

Fuente: propia 


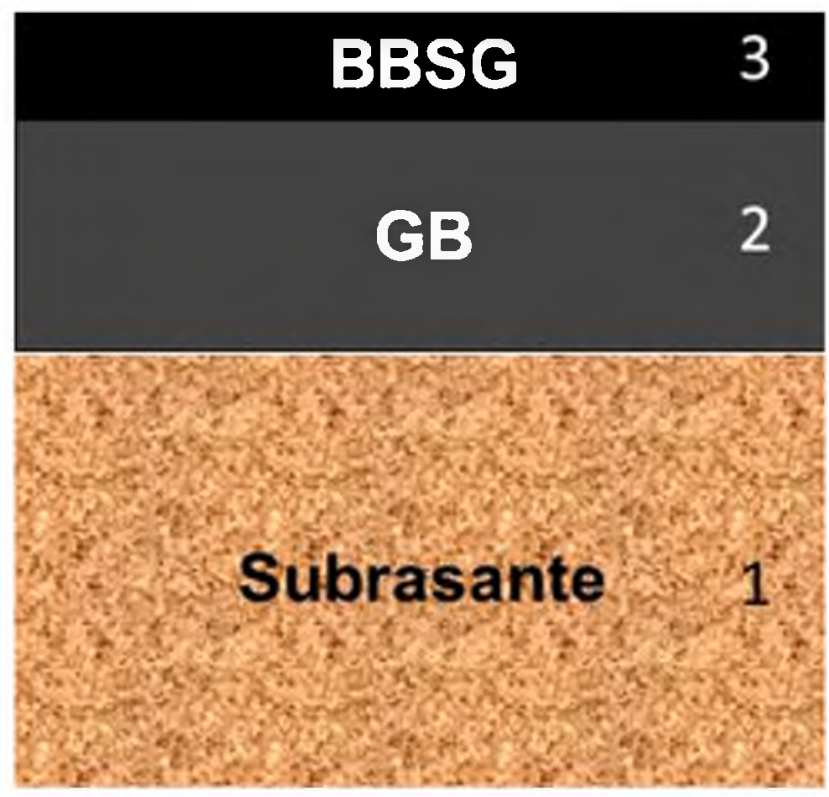

Figura 4. Estructura pavimento semi-flexible modelada. Fuente: propia

Tabla 2. Valores típicos de módulo elástico de las capas.

\begin{tabular}{rr}
\hline Capa & $\begin{array}{c}\text { Módulo elástico } \\
\text { (MPa) }\end{array}$ \\
\hline
\end{tabular}

Capa de rodadura

(concreto asfaltico BBSG)

5400

Base asfáltica (grava asfáltica GB)

9300

Subrasante

120

Fuente: propia.

como resiliente se determina por medio de ensayos triaxiales; el módulo elástico de las capas asfálticas se establece a través de ensayos de módulos resilientes ó dinámicos a temperaturas y frecuencias de diseño (LCPC, 1994), (AASHTO, 2008), (Kringos et al, 2013) o a través de tramos experimentales sobre los que se realizan ensayos deflectómetricos Falling Weight Deflectometer (FWD) ó Heavy Weight Deflectometer (HWD) acompañados de análisis inversos o de retrocalculo y corregidos a las condiciones de laboratorio (Papagiannakis, 2008), (Rabaiottia et al, 2013).

Ya que la variación del módulo de Poisson no tiene incidencia considerable en el comportamiento del pavimento (Rodríguez, 2005a), (Rodríguez, 2014b), se emplearon los valores característicos de la Tabla 3. Los espesores típicos, los módulos elásticos y las relaciones de Poisson fueron tomados de la Guía Francesa para el Diseño de Pavimentos Asfalticos por Metodología Racional "Conception et dimensionnement des structures de chaussée, Guide technique" del antiguo Laboratoire Central des Ponts et Chaussées (LCPC) (LCPC, 1994), hoy por hoy, Institut Français des Sciences et Technologies des Transports, de L'aménagement et des Réseaux (IFSTTARs), entidad de investigación reconocida a nivel mundial y líder en Europa.

Tabla 3. Valores típicos de módulo de Poisson de las capas constitutivas.

\begin{tabular}{lc}
\hline Capa & Módulo Poisson \\
\hline Capa de rodadura & \\
(concreto asfaltico BBSG) & 0.35 \\
Base asfáltica (grava asfáltica GB) & 0.35 \\
Subrasante & 0.35 \\
\hline
\end{tabular}

Fuente: propia

La determinación de los parámetros de diseño de pavimentos tienen principio en el diseño racional que consiste en modelar la estructura a partir de la definición de los espesores y las rigideces (módulos resilientes, dinámicos y de Poisson) de cada capa para calcular las tensiones y compresiones provocadas por una carga tipo e identificar: a) la deformación a tensión máxima $\left(\varepsilon_{t}\right)$ capaz de producir la rotura de las capas asfálticas; esta deformación se compara con el límite admisible del material asfáltico $\left(\varepsilon_{\text {tadm }}\right)$ que es función de múltiples factores, entre ellos, el tránsito, coeficientes de calibración y riesgo, efecto de las heterogeneidades locales de rigidez de las capas ligadas y deformación a un millón de solicitaciones con una probabilidad de falla del $50 \%$ determinado a $10^{\circ} \mathrm{C}$ y $25 \mathrm{~Hz}$ (LCPC, 1994); b) la deformación a compresión máxima $\left(\varepsilon_{\mathrm{c}}\right)$ sobre la subrasante que se compara con la admisible $\left(\varepsilon_{\text {cadm }}\right)$ y que depende del nivel de tránsito (LCPC, 1994).

\subsection{Modelo analítico EverStress $\bigcirc 5.0$}

Se desarrolló el modelo en el programa elástico multicapa de cálculo de esfuerzos y deformaciones para diseño de pavimentos EverStress(C) 5.0, introduciendo una presión de inflado distribuida uniformemente sobre un área circular de radio $0.125 \mathrm{~m}$ bajo una presión de $0.662 \mathrm{MPa}$ y distancia entre ejes de $0.375 \mathrm{~m}$. El análisis permite identificar las deformaciones características de control del diseño estructural del pavimento, tales como las deformaciones a tensión en las fibras inferiores de las capas asfálticas y compresión en la parte superior de la subrasante (LCPC, 1994). En la figura 5 se indica la ubicación de estos parámetros. 


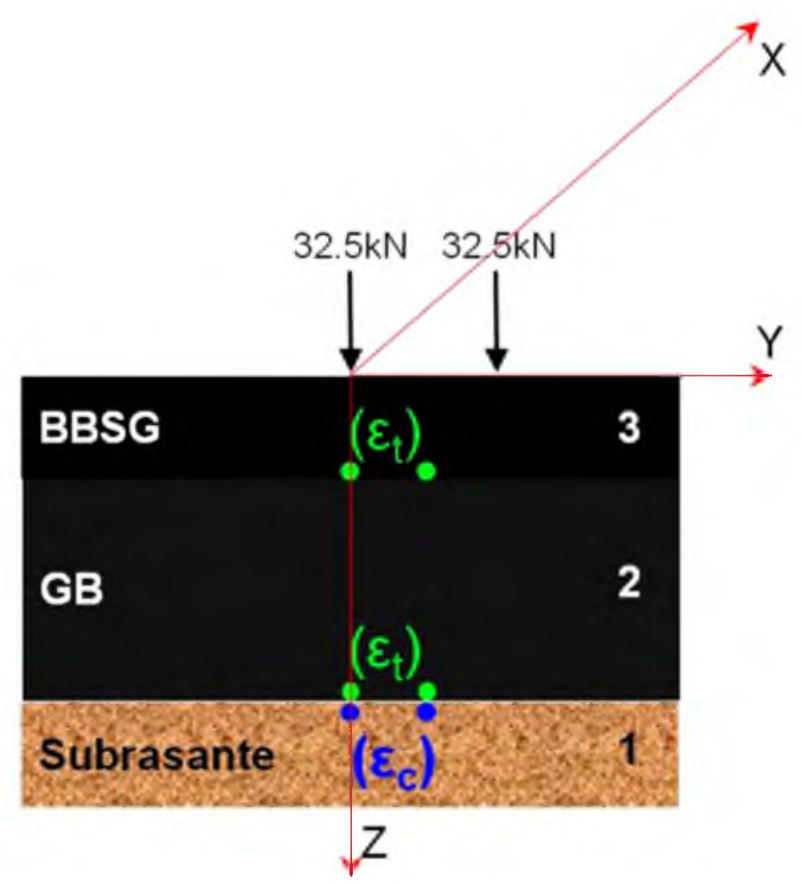

Figura 5. Ubicación de los parámetros de control en el diseño del pavimento EverStress $\mathbb{C}$ 5.0. Fuente: propia

\subsection{Modelos de elementos finitos EverStress FE 1.0}

Los modelos de elementos finitos con el modelador tridimensional elástico EverStressFE 1.0 fueron validados con el programa EverStress $($ C) 5. La figura 6 muestra el elemento finito que emplea el software; se trata de un elemento tipo BRICK con tres grados de libertad por nodo (UX, UY, UZ). El EverStressFE 1.0 trabaja con mallados de distintos grados de refinamiento y se compone de cinco interfaces que realizan diferentes funciones: a) Geometry and layer properties, contiene una herramienta CAD que permite construir un modelo geométrico e introducir los datos característicos de las capas (espesores y módulos) y definir las fronteras del dominio del modelo, b) Loads, almacena los parámetros de carga, tipo de rueday eje, contacto, presión, carga, ancho y espaciamiento de las llantas, distancia entre ejes y formas de distribución de la presión de contacto de la rueda, c) Meshing, determina los parámetros de mallado (tipo de malla y refinamiento, división de elementos, condición de interfaz y rigidez) y especificaciones del modelo (número de nodos y elementos finitos), d) Solver, ejecuta el solucionador de elementos finitos, e) Results, permite obtener los resultados del análisis. Los pasos típicos del procedimiento de análisis de un problema de esfuerzo-deformación con EverStressFE 1.0, son: ingresar los datos de dimensionamiento, numero de capas, espesores y parámetros de rigidez (módulos resilientes, dinámicos y de Poisson), condiciones de frontera, valores de presión, carga, ancho y espaciamiento de las

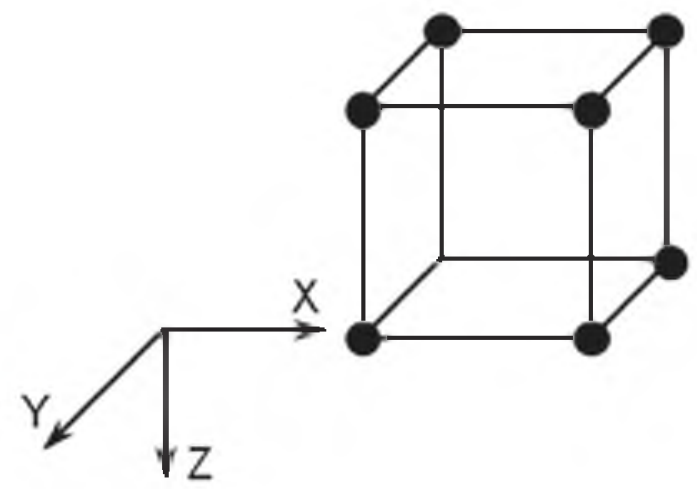

Figura 6. Elemento BRICK. Fuente: EverStressFE1.0 Theory Manual.

llantas (el programa por defecto aplica un cuarto de carga dual según se observa en las figuras 7,8 y 9), elegir una combinación de mallado, ejecutar el solucionador y obtener resultados (Davids, 2009a,b,c).

\subsubsection{Area de contacto y distribución de la presión}

Validado el modelo de elementos finitos con el área de contacto circular, se procedió a desarrollar tres modelos adicionales variando únicamente la distribución de la presión de contacto de la carga dual de manera más ajustada a la realidad (figuras 2 y 3 ). Los análisis se desarrollaron con los mismos parámetros de rigidez, espesores, distancia entre ejes y presión de contacto, teniendo en cuenta un área de huella rectangular cuyo ancho de banda "Tire Tread Width" se calcula a partir de las expresiones (1) y (2). La figura 10 justifica la procedencia del nuevo dato de cálculo.

$\mathrm{A}_{\text {circular }}=\pi \mathrm{r}^{2}=(\pi)(12.5 \mathrm{~cm})^{2}=490.87 \mathrm{~cm}^{2}$

$A_{\text {rectangular }}=490.87 \mathrm{~cm}^{2}=(5)(25)(x) \Rightarrow x=3.927 \mathrm{~cm}$

Donde $x$ es el ancho de banda de llanta, $A$ es el área de la huella y $r$ es el radio del área circular.

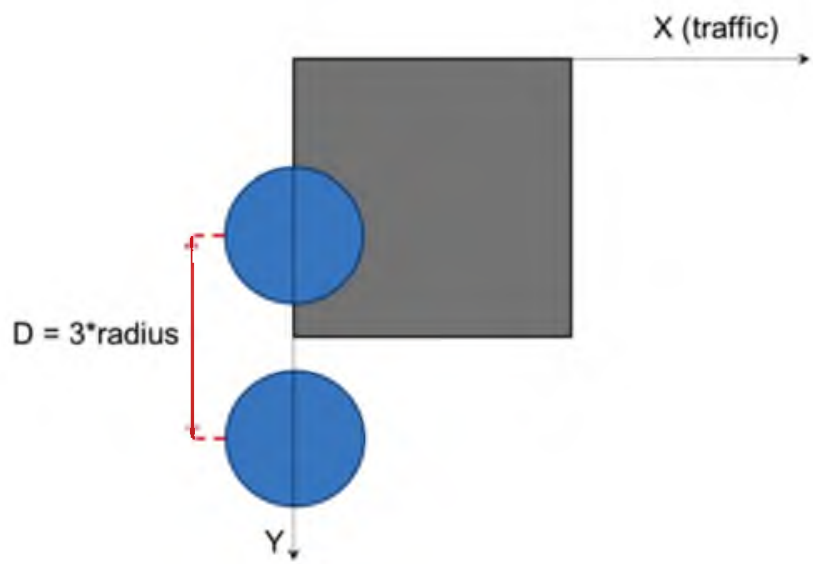

Figura 7. Vista en planta carga dual circular. Fuente: propia. 


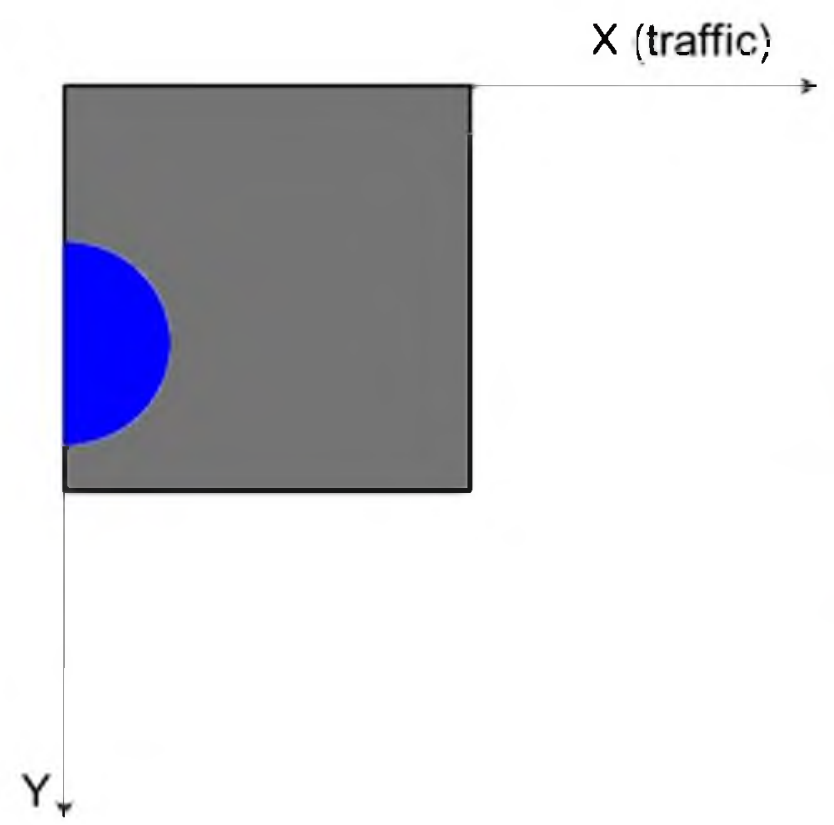

Figura 8. Vista en planta cuarto de carga dual circular. Fuente: propia.

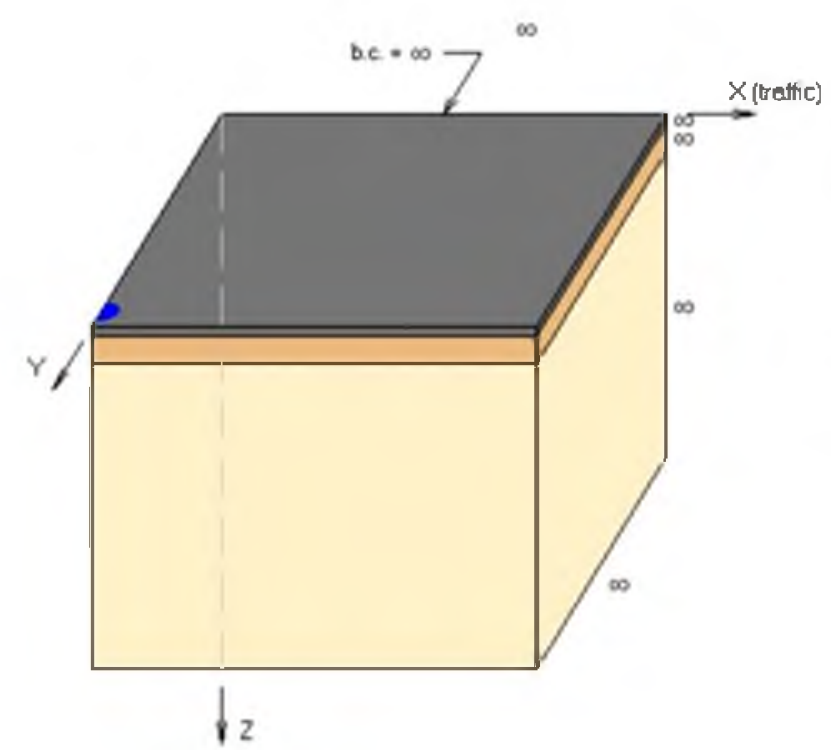

Figura 9. Vista 3D cuarto de carga dual circular. Fuente: propia.

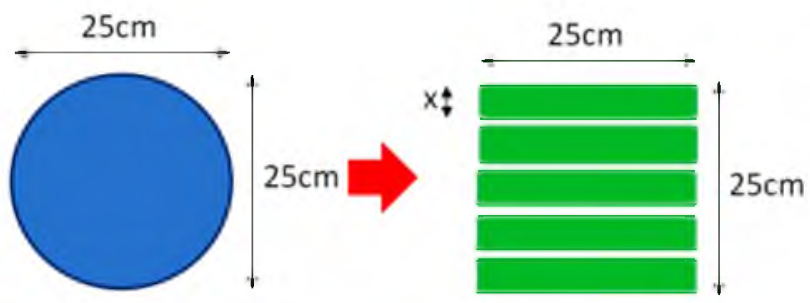

Figura 10. Área de contacto circular y huella rectangular. Fuente: propiaki.
Definida la forma de la huella, se evaluaron diferentes distribuciones de la presión de contacto: constante, parabólica y media onda sinusoidal como se muestra en las figuras 11,12 y 13 ; destacando que el pulso sinusoidal

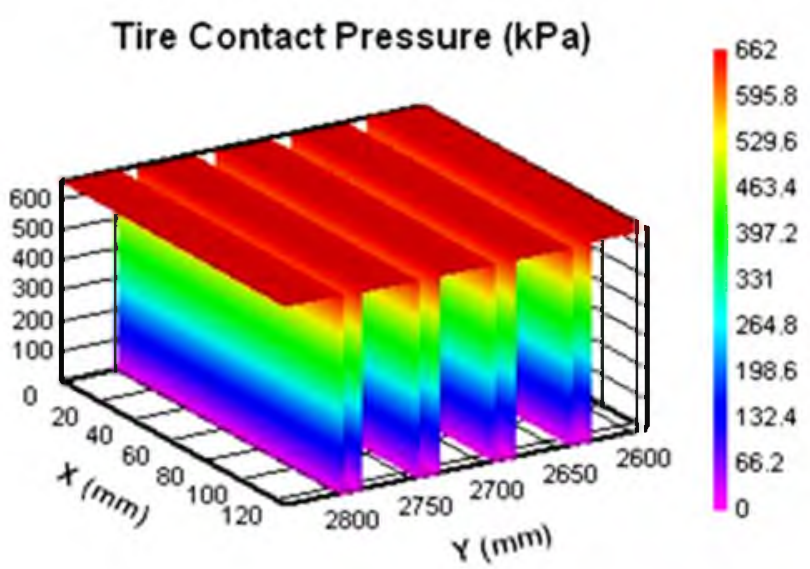

Figura 11. Distribución a lo largo de la llanta presión constante. Fuente: propia.

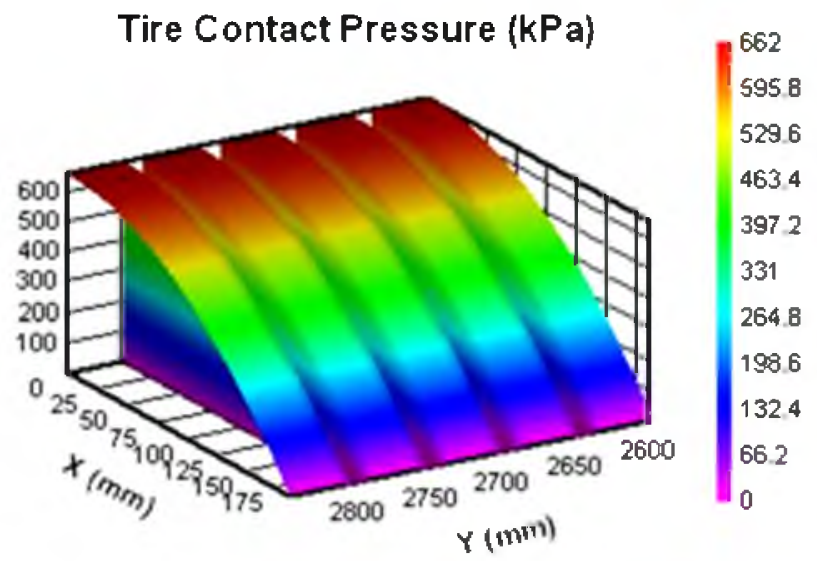

Figura 12. Distribución a lo largo de la llanta presión parabólica. Fuente: propia.

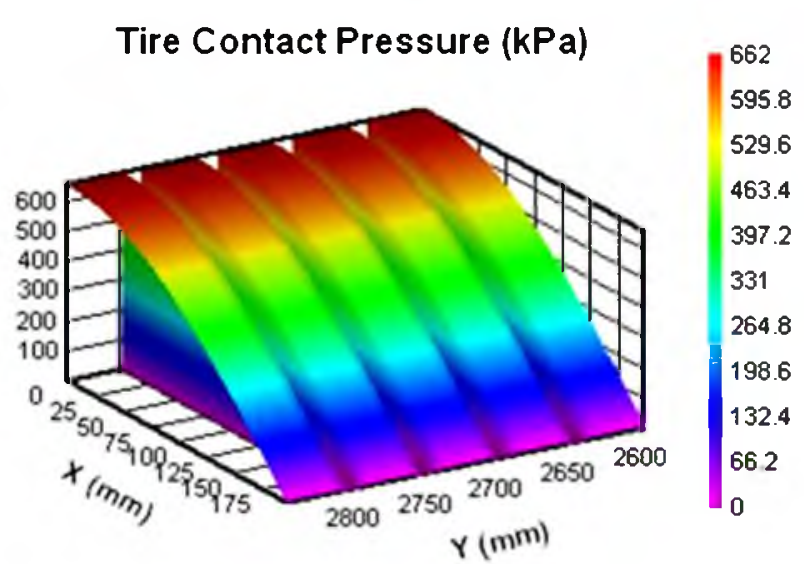

Figura 13. Distribución a lo largo de la llanta presión media onda sinusoidal. Fuente: propia. 
es característico en la determinación de la curva maestra de ensayos dinámicos de concreto asfaltico, de hecho, la prueba de módulo dinámico mide la relación esfuerzo-deformación de una muestra bajo un pulso de carga sinusoidal continuo (Brown et al, 2001), (Witczak et al, 2002), (Bonaquist, 2005). Al igual que en el caso de área de contacto circular, el programa ubica la carga dual a un cuarto (1/4-simétrica) para optimizar el tiempo de cálculo (Davids, 2009a, b, c). Las ilustraciones del caso se presentan en las figuras 14, 15 y 16.

\section{Resultados y discusión}

En la Tabla 4 se presentan los resultados del modelo analítico realizado con EverStress(C) 5.0 - utilizado como validador - y del modelo de elementos fínitos Ever StressFE 1.0 trabajado con área de contacto circular.

Se observa que los porcentajes de variación del modelo numérico frente al analítico son inferiores al $1 \%$ lo cual permite concluir que las decisiones tomadas en la modelación con EverStressFE 1.0 son adecuadas para implementar los modelos que introducen modificacio-

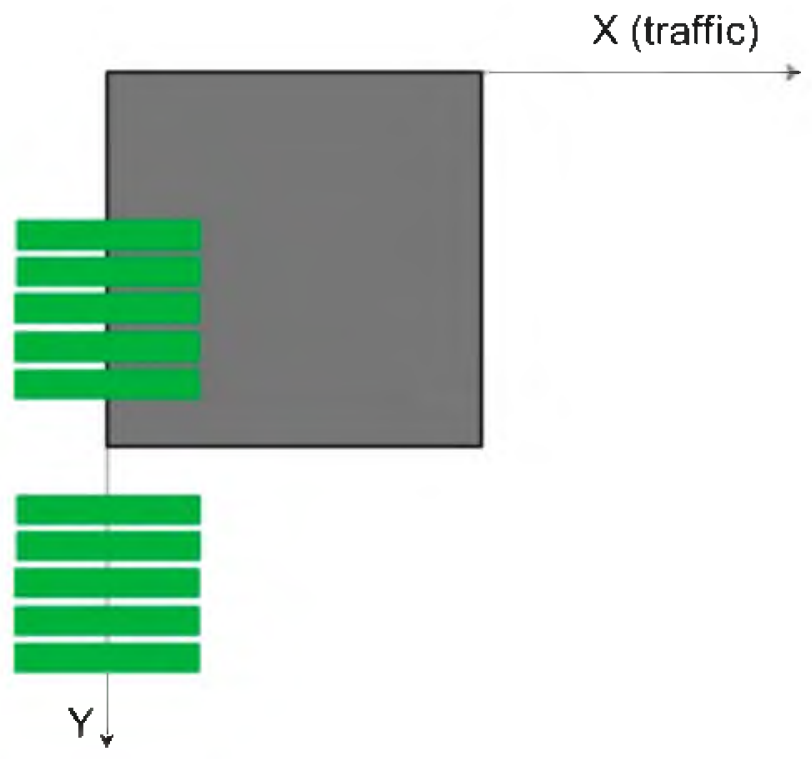

Figura 14. Vista en planta carga dual rectangular. Fuente: propia. nes en la forma de la huella y la presión de contacto de la llanta. En las figuras 17 y 18 se presentan los resultados del modelo analítico; los valores subrayados corresponden a los parámetros del diseño racional.

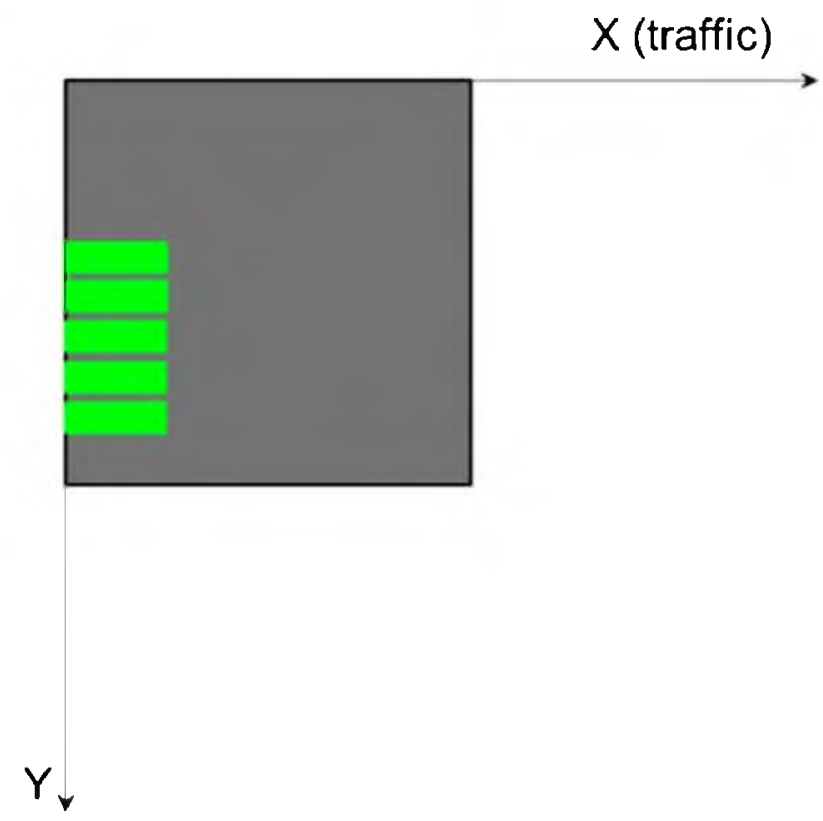

Figura 15. Vista en planta cuarto de carga dual rectangular. Fuente: propia.

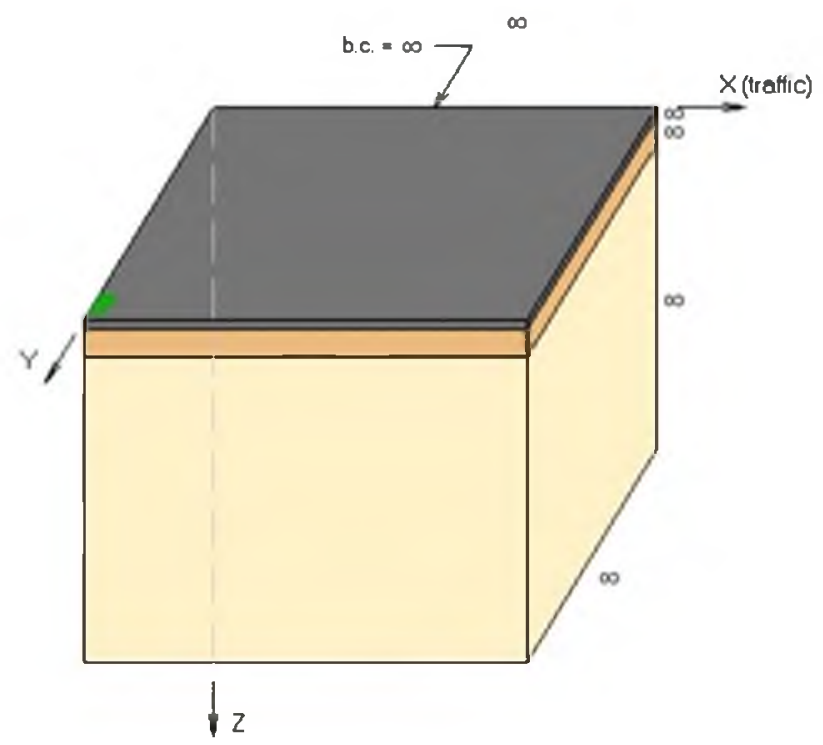

Figura 16. Vista tridimensional cuarto de carga dual rectangular. Fuente: propia.

Tabla 4. Validación de los resultados de control de diseño estructural de un pavimento semi-flexible.

\begin{tabular}{c|c|c|c|c}
\hline Capa & Parámetro & Analítico & Elementos Finitos 3D & \%Variación \\
\hline BBSG & $\varepsilon \times x \rightarrow \varepsilon c$ & $-38.81 \mathrm{E}-06$ & $-38.83 \mathrm{E}-06$ & $0.05 \%$ \\
\hline GB & $\varepsilon \times x \rightarrow \varepsilon t$ & $84.26 \mathrm{E}-06$ & $84.27 \mathrm{E}-06$ & $0.01 \%$ \\
\hline Subrasante & $\varepsilon \mathrm{zZ} \rightarrow \varepsilon \mathrm{c}$ & $-264.94 \mathrm{E}-06$ & $-262.96 \mathrm{E}-06$ & $0.75 \%$ \\
\hline
\end{tabular}

Fuente: propia. 


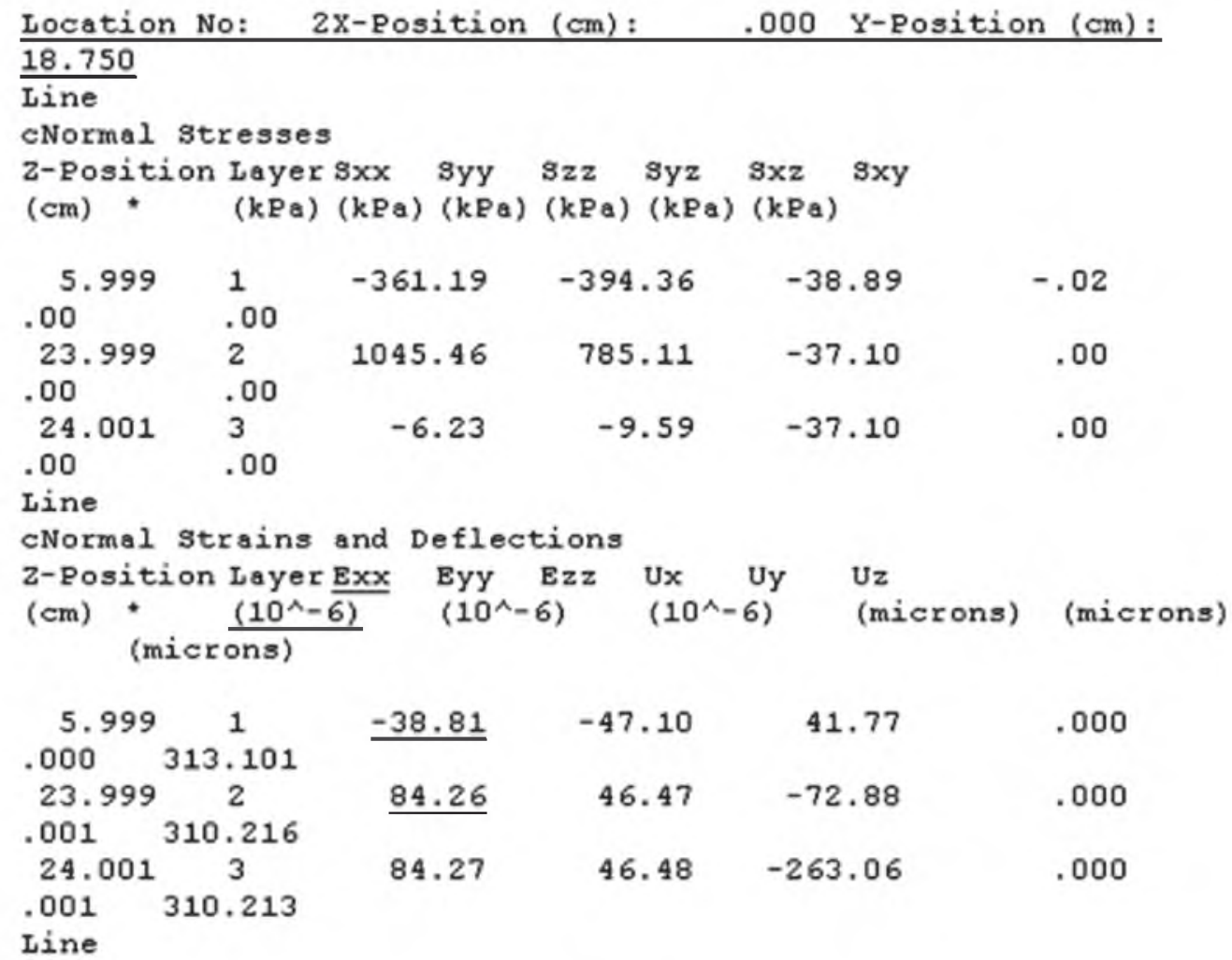

Figura 17. Resultados de deformación exx en las fibras inferiores de las capas asfálticas BBSG y GB en EverStress (C) 5.0 con presión constante circular. Fuente: propia.

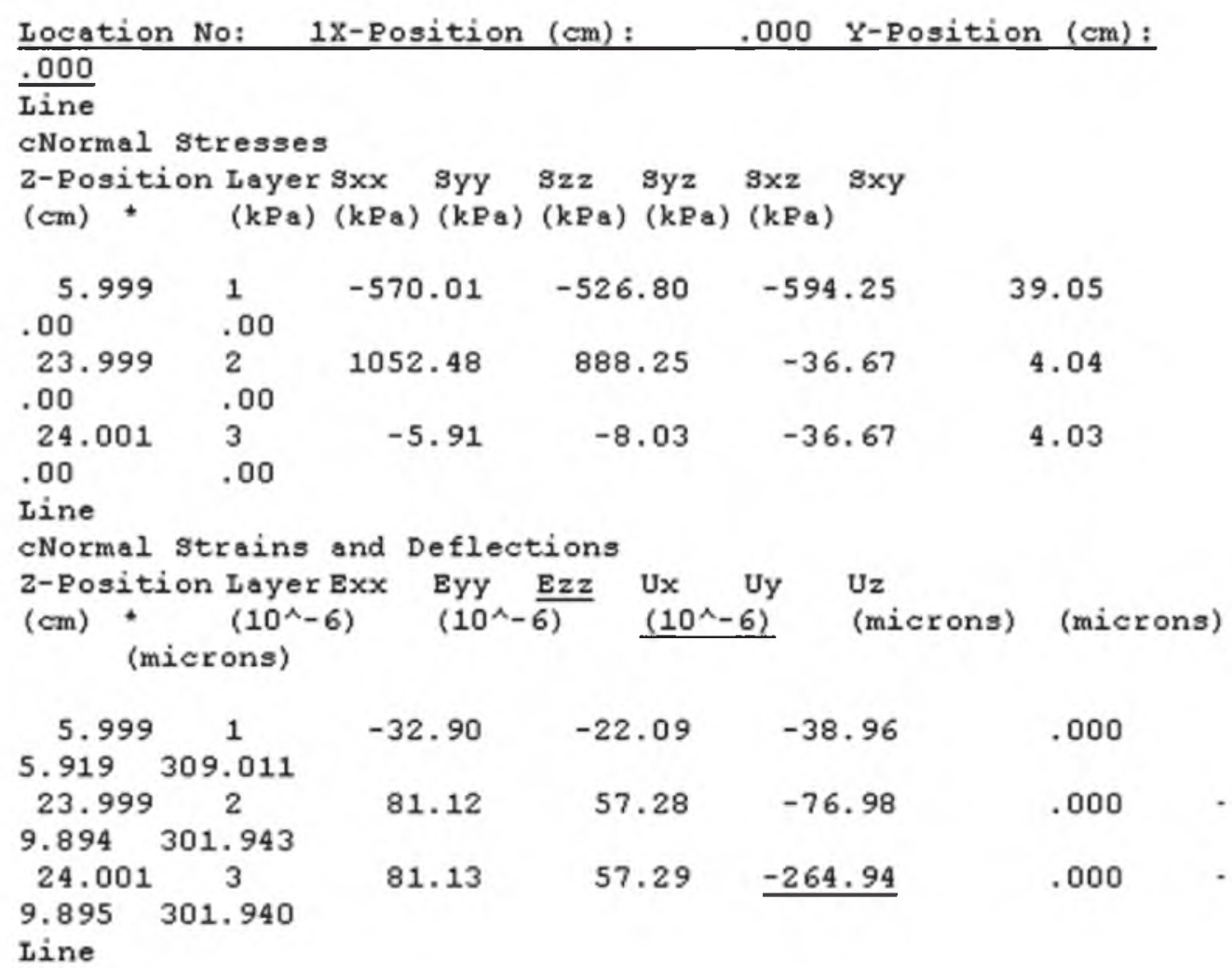

Figura 18. Resultados de deformación દzz sobre la subrasante en EverStress $\mathbb{C} 5.0$ con presión constante circular. Fuente: propia. 
De los resultados contenidos en las figuras 17 y 18 se verifica que: 1) la deformación máxima a compresión sobre la subrasante en el modelo multicapa elástico está bajo la llanta y no en el centro de la carga dual, 2) las deformaciones máximas en las fibras inferiores de las capas asfálticas BBSG y GB están bajo el centro de la carga dual y no bajo la llanta.

Las figuras 19,20 y 21 muestran los resultados gráficos de deformación, correspondientes a los parámetros del diseño racional por medio de isocontornos. La figura 19 representa las deformaciones $\varepsilon x x$ situadas en la fibra inferior de la capa asfáltica BBSG se observa la concentración de esfuerzos en la zona de aplicación de la carga. El semicírculo corresponde a un cuarto de carga dual con la forma típica de la huella empleada por el método racional. Los valores de deformación Exx mostrados en la figura 19 , sugieren un comportamiento a compresión de la capa asfáltica inclusive en la fibra inferior lo cual probablemente es atribuible al módulo de la capa asfáltica de rodadura BBSG a la rigidez de la capa contigua GB y a la misma condición de liga de las interfaces. El valor de deformación máxima Exx en esta capa se sitúa en el centro de la carga dual (coordenadas: $X=0, Y=2900$ ) tal como lo sugiere el modelo multicapa.

La figura 20 representa las deformaciones exx situadas en la fibra inferior de la capa asfáltica GB. Los

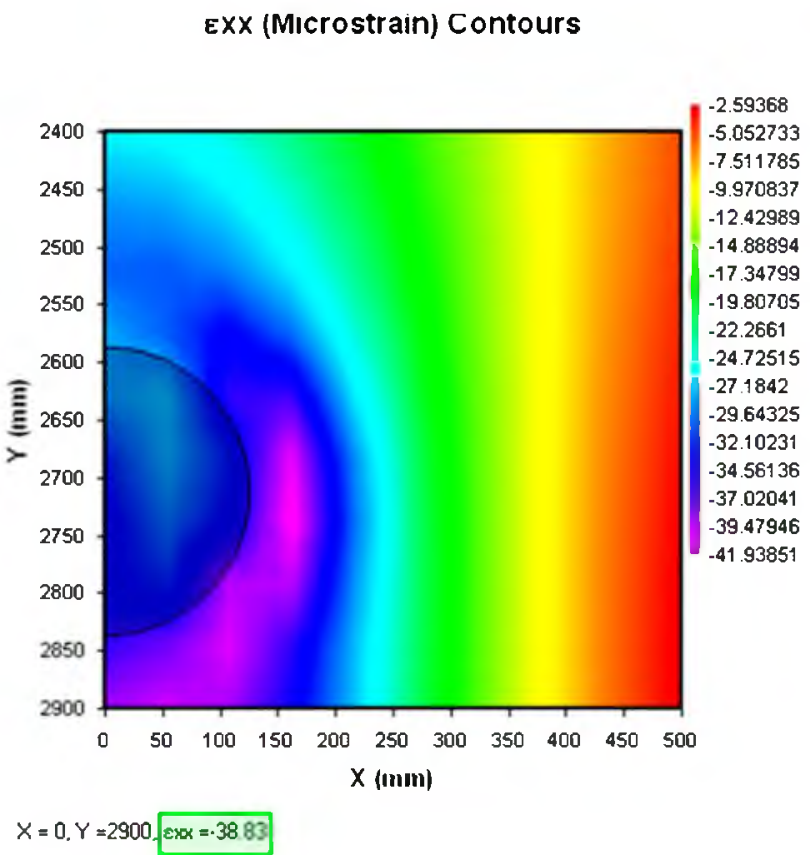

Figura 19. Deformación exx en la fibra inferior de la capa asfáltica BBSG en EverStressFE 1.0 con presión constante circular. Fuente: propia.
Exx (Microstrain) Contours

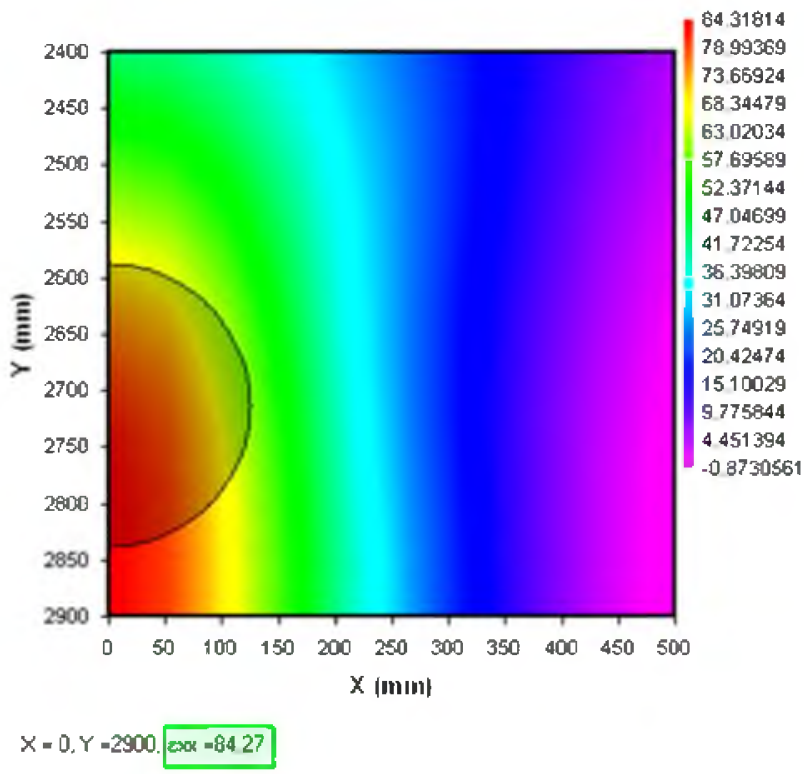

Figu ra 20. Deformación $\varepsilon x x$ en la fibra inferior de la capa asfáltica GB en EverStressFE 1.0 con presión constante circular. Fuente: propia.

$\varepsilon z z$ (Microstrain) Contours

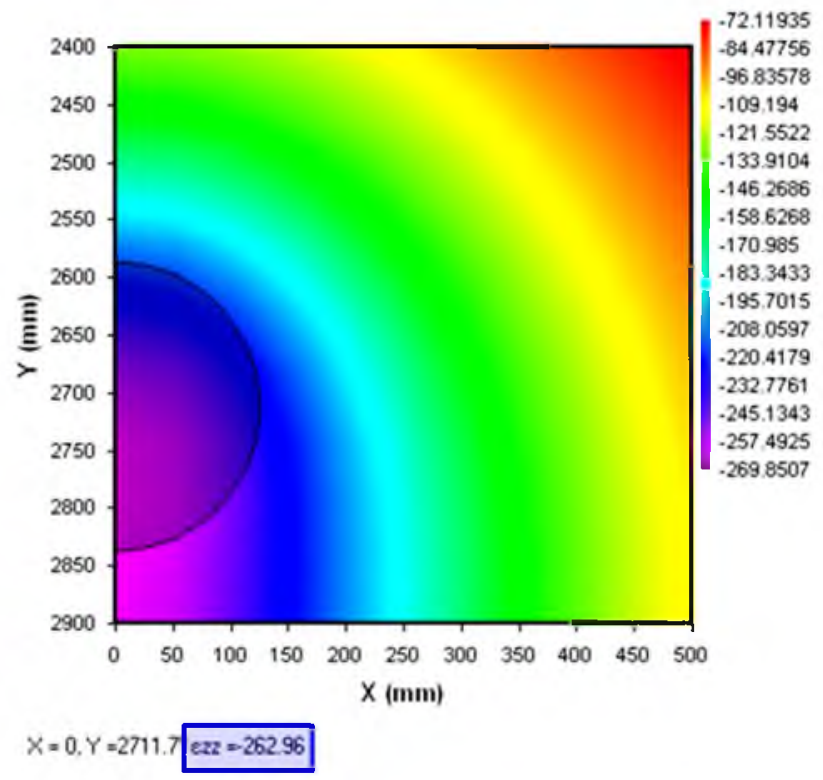

Figura 21. Deformación Ezz sobre la subrasante en EverStressFE 1.0 con presión constante circular. Fuente: propia.

valores de deformación mostrados en la figura, denotan el típico comportamiento a tensión de la capa asfáltica como lo sugiere el método racional en el control de diseño. El valor de deformación máxima Exx 
en esta capa se sitúa en el centro de la carga dual (coordenadas: $\mathrm{X}=0, \mathrm{Y}=2900$ ) tal como lo indica el modelo multicapa.

Los isocontornos de deformación vertical de la figura 21 siguen el patrón de la huella circular, encontrándose que el valor de deformación máxima vertical Ezzen esta capa se sitúa en el centro de la carga dual (coordenadas: $X=0, Y=2900$ ) y no en el centro de la llanta como lo sugiere el modelo multicapa, lo cual probablemente es debido a la carga dual y/o a la misma rigidez de la estructura y de la subrasante.

\subsection{Modelos de elementos finitos con área de contacto rectangular y otras distribuciones de presión}

Se desarrollaron tres modelos para analizar la respuesta a la deformación de la estructura de pavimento cuando se emplea un área de contacto y una distribución de la presión de inflado de las llantas sobre la superficie de manera más ajustada a la realidad. El área de contacto corresponde a la huella típica rectangular mostrada en la figura 3 y se evaluaron las distribuciones de presión constante, parabólica y media onda sinusoidal de las figuras 11,12 y 13 respectivamente.

\subsubsection{Presión constante}

La figura 22 presenta el desarrollo de la deformación Exx en la fibra inferior de la capa asfáltica BBSG con presión de inflado constante. En el centro de la carga dual ubicado en las coordenadas $X=0, Y=2900$ y Z $=60$ $\mathrm{cm}$ se encuentra la máxima deformación normal $\mathrm{Exx}=$ 39.77E-06 (-39.77 microstrain). Esta deformación por ser de compresión se considera no representativa en el diseño y señala que el eje neutro de la estructura se encuentra por debajo de la capa de rodadura BBSG En adelante el análisis de resultados se desarrollará respecto al modelo de referencia de presión constante y área de contacto circular. Al comparar los resultados de isocontornos de deformación Exx con los del modelo de referencia, se observa que el rango de valores de deformación es similar, al igual que la distribución de los isocontornos en el plano. Las deformaciones promedio de mayor valor se encuentran capturadas dentro de la huella rectangular. La distribución de la deformación indica que la huella rectangular es una buena representación del contacto de la llanta con la superficie del pavimento.

La figura 23 presenta los resultados de la deformación åxx en la fibra inferior de la capa asfáltica GB. Entendiendo al pavimento como un elemento sometido a flexión, $\varepsilon \times x$ (Microstrain) Contours

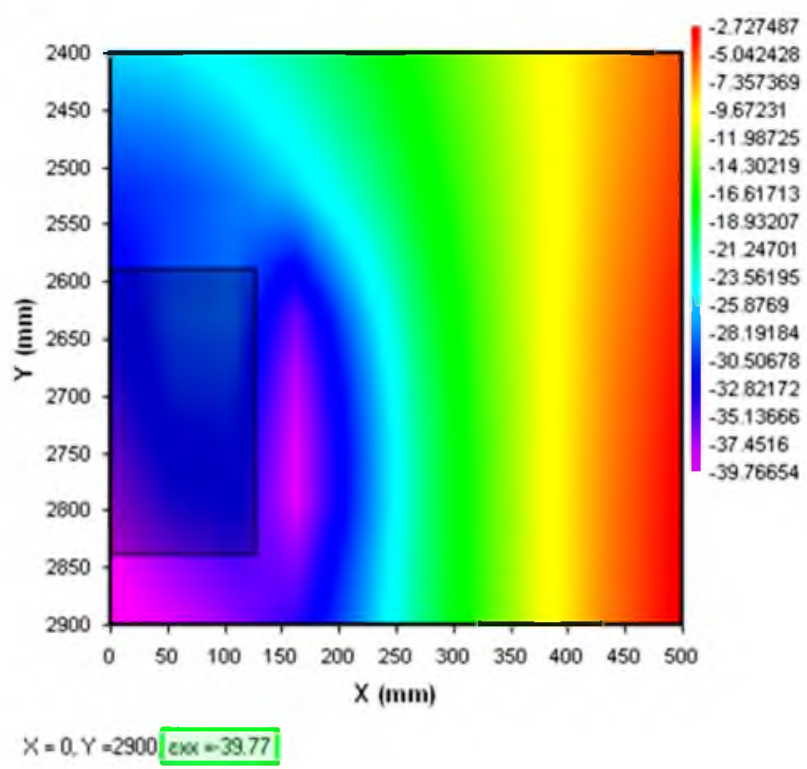

Figura 22. Deformación exx en la fibra inferior de la capa asfáltica BBSG en EverStressFE 1.0 con presión constante rectangular. Fuente: propia.

ExX (Microstrain) Contours

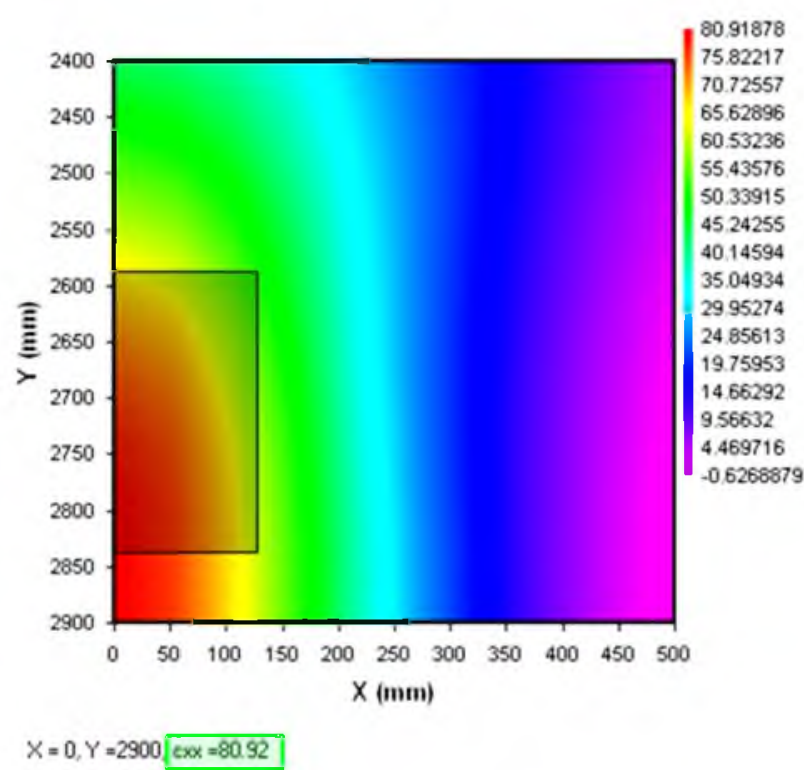

Figu ra 23. Deformación exx en la fibra inferior de la capa asfáltica GB en EverStressFE 1.0 con presión constante rectangular. Fuente: propia.

se aprecia que el eje neutro de la estructura se encuentra dentro del dominio de esta capa. La máxima deformación normal Exx es 80.92E-06 (80.92 microstrain), está ubicada en el centro de la carga dual con coordenadas $X=0, Y=2900$, pero con $Z=240 \mathrm{~cm}$ y representa el estado de tensión característico del diseño racional. 
La figura 24 representa la deformación $\varepsilon z z$ sobre la subrasante. La máxima deformación normal $\varepsilon z z$ de 253.27E-06 (-253.27 Microstrain) está ubicada en las coordenadas $X=0, Y=2900$ y $Z=240 \mathrm{~cm}$.

La Tabla 5 contiene el resumen de los resultados comparados con los obtenidos del modelo elástico multicapa EverStress 5.0, determinando porcentajes de variación bajos y poco significativos.

\subsubsection{Presión parabólica}

La figura 25 indica la deformación exx en la fibra inferior de la capa asfáltica BBSG Al comparar los resultados de isocontornos de deformación Exx con los del modelo de referencia, se observa que el rango de valores de deformación es similar, no obstante, la distribución de los isocontornos en el plano es ligeramente diferente, esto es, el contorno de deformación máxima se concentra en el centro de la carga dual, significando menor densidad de deformación normal a compresión alrededor de la huella.

$\varepsilon z z$ (Microstrain) Contours

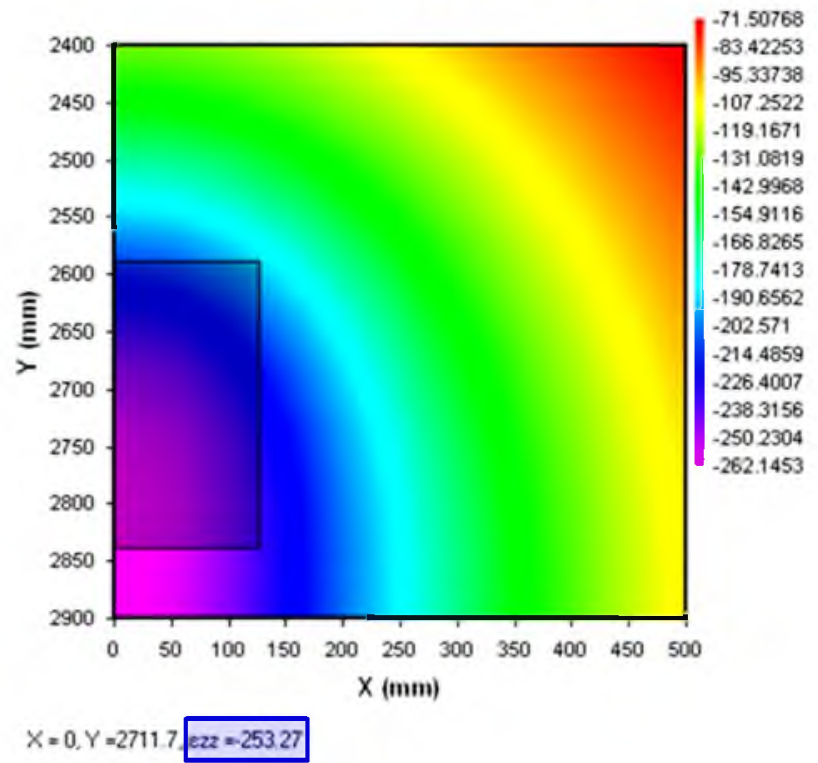

Figura 24. Deformación Ezz sobre la subrasante en EverStressFE 1.0 con presión constante rectangular. Fuente: propia. $\operatorname{exx}$ (Microstrain) Contours

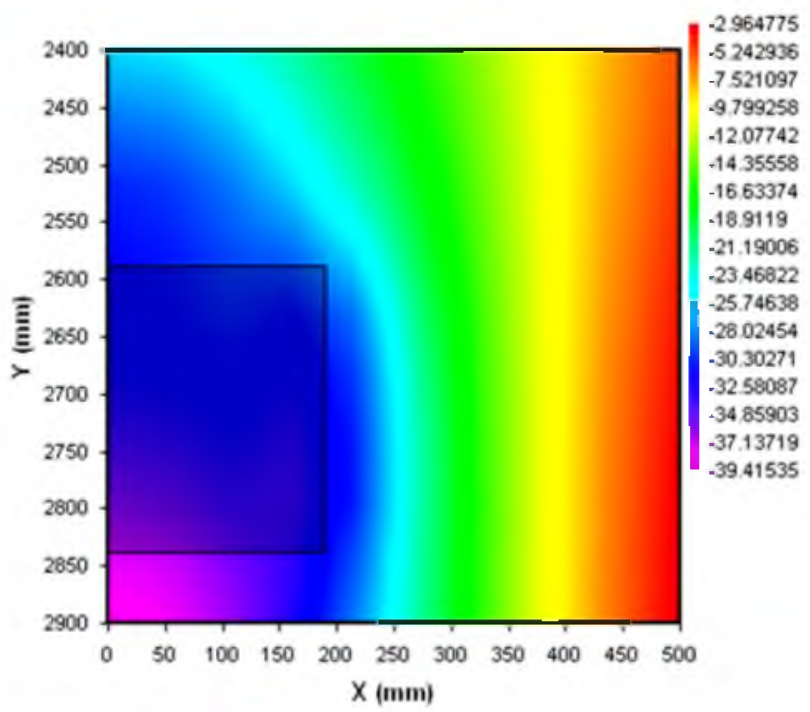

$X=0 . Y=2900,000-39.42$

Figura 25. Deformación $\varepsilon x x$ en la fibra inferior de la capa asfáltica BBSG en EverStressFE 1.0 con presión parabólica rectangular. Fuente: propia.

En la figura 26 se presenta la deformación exx en la fibra inferior de la capa asfáltica GB. La máxima deformación normal Exx es 78.95E-06 (78.95 microstrain) y se ubica en las coordenadas $X=0$, $\mathrm{Y}=2900$ y $\mathrm{Z}=240 \mathrm{~cm}$.

La figura 27 reproduce la deformación Ezz sobre la subrasante. En la coordenada X=0, Y=2900 y Z $=240$ $\mathrm{cm}$ se ubica la máxima deformación normal a compresión Ezz de -251.67E-06 (-251.67 microstrain). Al comparar los resultados de isocontornos de deformación Ezz de la figura 27 con los del modelo de referencia, se observa que el isocontorno de deformación máxima es más amplio denotando un mayor rango de afectación a compresión. Adicionalmente, dentro de la huella se desarrollan más isocontornos denotando menos homogeneidad o uniformidad de deformación dentro de la misma.

La tabla 6 contiene el resumen de los resultados comparados con los obtenidos del modelo elástico

Tabla 5. Variación de los parámetros de control de diseño estructural de un pavimento semi-flexible mediante una presión constante rectangular.

\begin{tabular}{c|c|c|c|c}
\hline Capa & Parámetro & Analítico & Elementos Finitos 3D & \%Variación \\
\hline BBSG & $\varepsilon x x \rightarrow \varepsilon c$ & $-38.81 \mathrm{E}-06$ & $-39.77 \mathrm{E}-06$ & $2.47 \%$ \\
\hline GB & $\varepsilon x x \rightarrow \varepsilon t$ & $84.26 \mathrm{E}-06$ & $80.92 \mathrm{E}-06$ & $3.96 \%$ \\
\hline Subrasante & $\varepsilon \mathrm{zZ} \rightarrow \varepsilon \mathrm{c}$ & $-264.94 \mathrm{E}-06$ & $-253.27 \mathrm{E}-06$ & $4.40 \%$ \\
\hline
\end{tabular}

Fuente: propia. 
Tabla 6. Variación de los parámetros de control de diseño estructural de un pavimento semi-flexible mediante una presión parabólica rectangular.

\begin{tabular}{c|c|c|c|c}
\hline Capa & Parámetro & Analítico & Elementos Finitos 3D & \%Variación \\
\hline BBSG & $\varepsilon x x \rightarrow \varepsilon c$ & $-38.81 \mathrm{E}-06$ & $-39.42 \mathrm{E}-06$ & $1.57 \%$ \\
\hline GB & $\varepsilon \mathrm{xx} \rightarrow \varepsilon \mathrm{ct}$ & $84.26 \mathrm{E}-06$ & $78.95 \mathrm{E}-06$ & $6.30 \%$ \\
\hline Subrasante & $\varepsilon \mathrm{zz} \rightarrow \varepsilon \mathrm{c}$ & $-264.94 \mathrm{E}-06$ & $-251.67 \mathrm{E}-06$ & $5.01 \%$ \\
\hline
\end{tabular}

Fuente: propia.

$\varepsilon \times \times$ (Microstrain) Contours

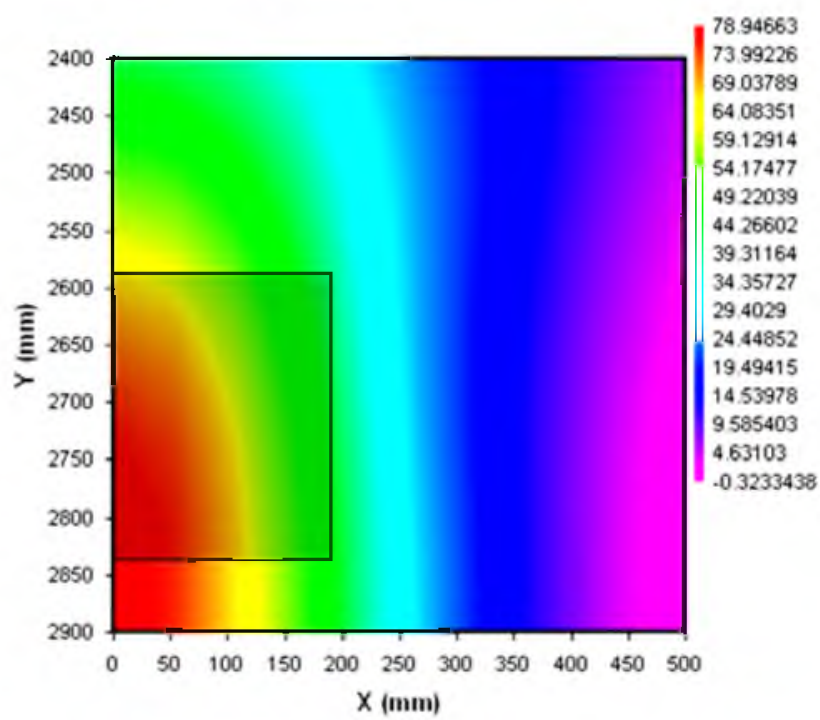

$X=0 . Y=29000000=78.95$

Figura 26. Deformación exx en la fibra inferior de la capa asfáltica GB en EverStressFE 1.0 con presión parabólica rectangular. Fuente: propia.

multicapa EverStress 5.0. Este resultado llama la atención porque las deformaciones $\varepsilon x x$ y $\varepsilon z z$, son variables representativas del diseño y los porcentajes de variación sugieren posibles sobredimensionamientos, por consiguiente mayores costos de la estructura.

\subsubsection{Presión media onda sinusoidal}

La figura 28 nos muestra el mapa de colores de la deformación Exx en la fibra inferior de la capa asfáltica BBSG La máxima deformación normal Exx es -39.30E-06 (-39.30 microstrain) y se encuentra en las coordenadas $\mathrm{X}=0, \mathrm{Y}=2900 \mathrm{y} \mathrm{Z}=60 \mathrm{~cm}$.

La figura 29 expone el mapa de colores de la deformación Exx en la fibra inferior de la capa asfáltica. En las coordenadas $X=0, Y=2900$ y $Z=240 \mathrm{~cm}$ se presenta la máxima deformación normal $\varepsilon x x$ de $78.61 \mathrm{E}-06$ (78.61 microstrain) $\varepsilon z z$ (Microstrain) Contours

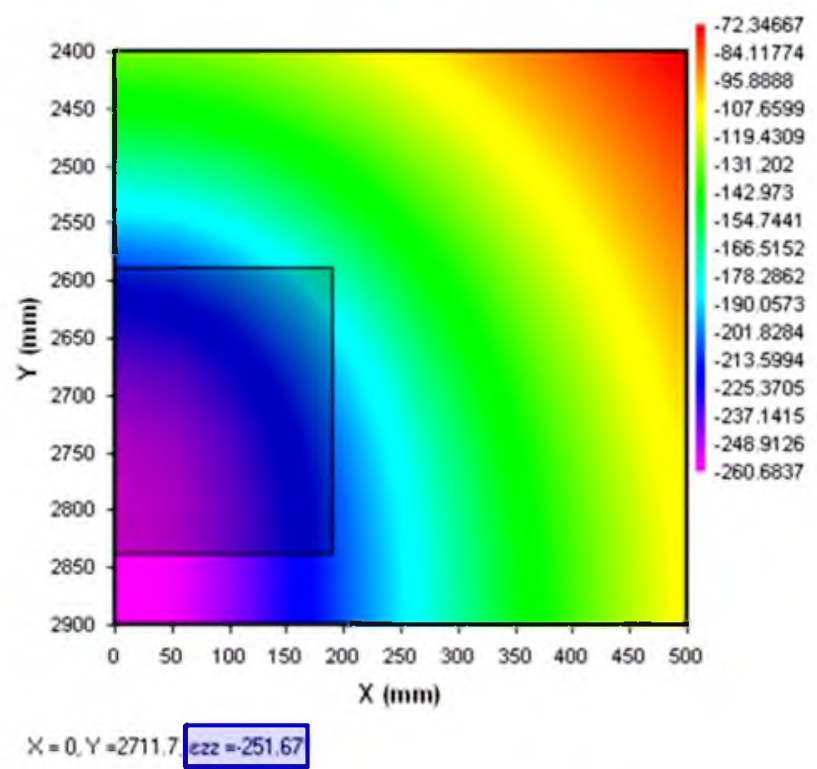

Figura 27. Deformación Ezz sobre la subrasante en Ever StressFE 1.0 con presión parabólica rectangular. Fuente: propia.

\section{EXX (Microstrain) Contours}

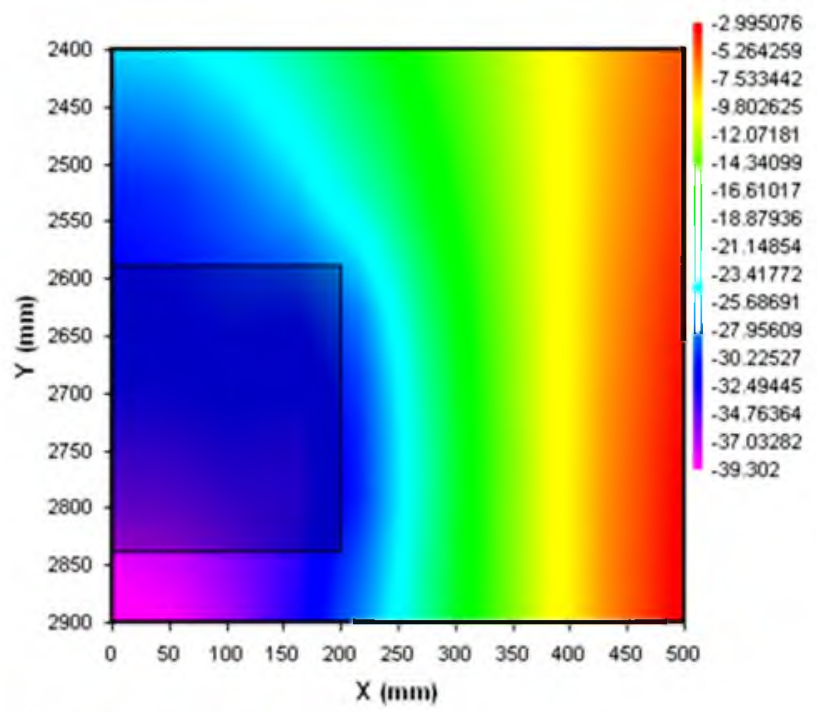

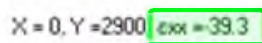

Figu ra 28. Deformación exx en la fibra inferior de la capa asfáltica BBSG en EverStressFE 1.0 con presión media onda sinusoidal. Fuente: propia. 
ExX (Microstrain) Contours

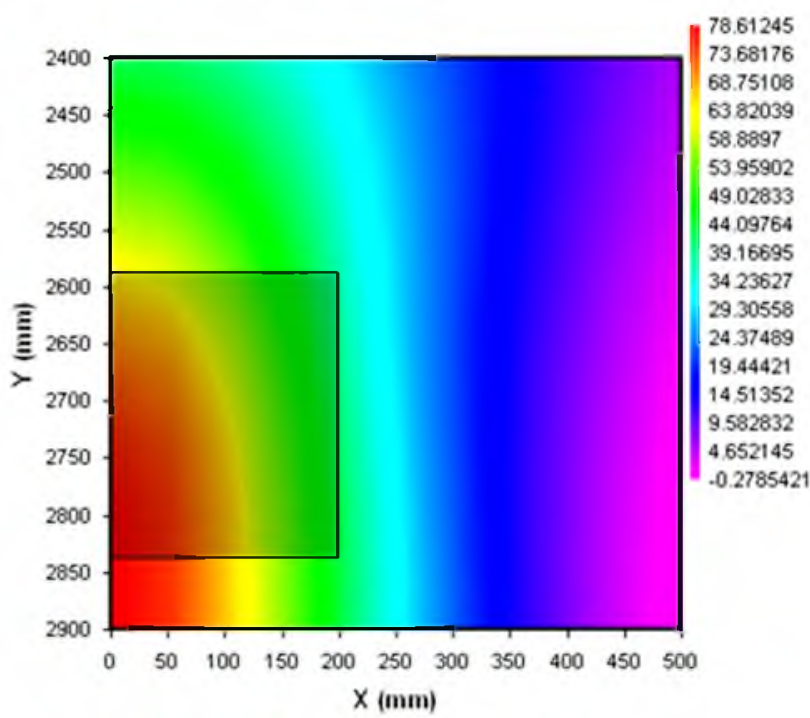

$X=0, Y=2900$ exx $=78.61$

Figura 29. Deformación exx en la fibra inferior de la capa asfáltica GB en EverStressFE 1.0 con presión media onda sinusoidal. Fuente: propia

En la figura 30 se presenta el comportamiento de la deformación $\varepsilon z z$ sobre la subrasante. La máxima deformación normal $\varepsilon z Z$ es -251.18E-06 (-251.18 microstrain) y se ubica en las coordenadas $X=0$, $\mathrm{Y}=2900 \mathrm{y} \mathrm{Z}=240 \mathrm{~cm}$.

La tabla 7 presenta el resumen de los resultados comparados con los valores obtenidos del modelo elástico multicapa EverStress 5.0, encontrándose porcentajes de $\varepsilon z z$ (Microstrain) Contours

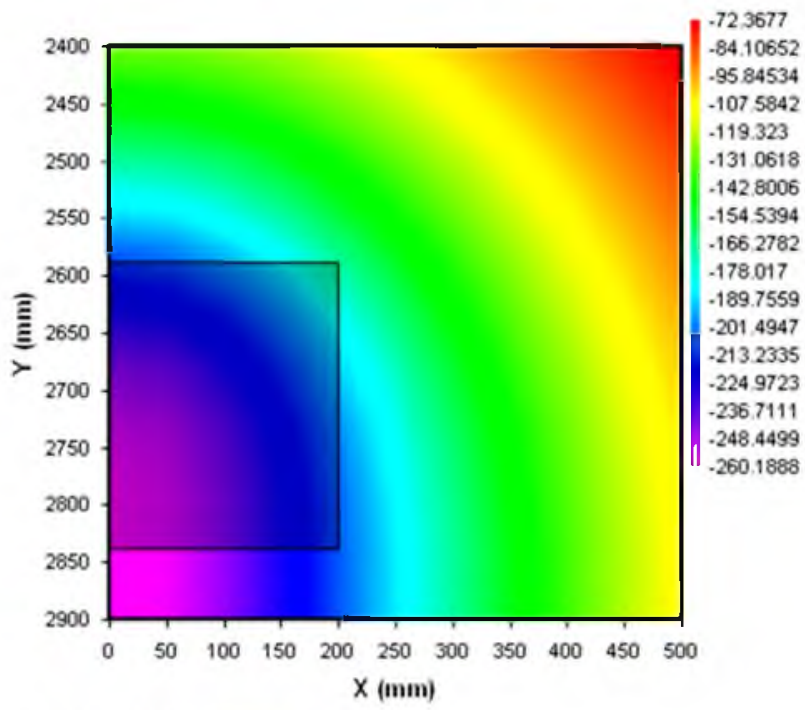

$X=0, Y=2711.7 \quad e z 2-251.18$

Figura 30. Deformación ezz sobre la subrasante en EverStressFE 1.0 con presión media onda sinusoidal. Fuente: propia.

variación de $1.26 \%$ y $6.71 \%$ para las capas asfálticas y $5.19 \%$ sobre la subrasante. Este escenario también llama la atención, porque las deformaciones $\varepsilon x x$ y $\varepsilon z z$ determinadas por el método analítico se encuentran 6.30 y $5.01 \%$ por encima de los resultados del modelo de elementos finitos, sugiriendo sobredimensionamiento de la estructura. Probablemente, el diseño racional debería ajustarse a condiciones más reales de área de contacto y distribución de presión.

Tabla 7. Variación de los parámetros de control de diseño estructural de un pavimento semi-flexible mediante una presión media onda sinusoidal rectangular.

\begin{tabular}{c|c|c|c|c}
\hline Capa & Parámetro & Analítico & Elementos Finitos 3D & \%Variación \\
\hline BBSG & $\varepsilon x x \rightarrow \varepsilon c$ & $-38.81 \mathrm{E}-06$ & $-39.30 \mathrm{E}-06$ & $1.26 \%$ \\
\hline GB & $\varepsilon x x \rightarrow \varepsilon t$ & $84.26 \mathrm{E}-06$ & $-25.61 \mathrm{E}-06$ & $6.71 \%$ \\
\hline Subrasante & $\varepsilon \mathrm{ZZ} \rightarrow \varepsilon \mathrm{c}$ & $-264.94 \mathrm{E}-06$ & $-251.18 \mathrm{E}-06$ & $5.19 \%$ \\
\hline
\end{tabular}

Fuente: propia

Tabla 8. Resumen de parámetros de control de diseño estructural del pavimento semi-flexible.

\begin{tabular}{c|c|c|c|c|c}
\hline \multirow{2}{*}{ Capa } & Parametro & \multirow{2}{*}{ Analítico } & \multicolumn{3}{|c}{ elementos finitos 3d } \\
\cline { 4 - 6 } & & & $\begin{array}{c}\text { Presión } \\
\text { constante }\end{array}$ & $\begin{array}{c}\text { Presión } \\
\text { parabólica }\end{array}$ & $\begin{array}{c}\text { Presión media onda } \\
\text { sinusoidal }\end{array}$ \\
\hline $\mathrm{BBSG}$ & $\varepsilon \mathrm{xx} \rightarrow \varepsilon \mathrm{c}$ & $-38.81 \mathrm{E}-06$ & $-39.77 \mathrm{E}-06$ & $-39.42 \mathrm{E}-06$ & $-39.30 \mathrm{E}-06$ \\
\hline $\mathrm{GB}$ & $\varepsilon \mathrm{xx} \rightarrow \mathrm{ct}$ & $84.26 \mathrm{E}-06$ & $80.92 \mathrm{E}-06$ & $78.95 \mathrm{E}-06$ & $78.61 \mathrm{E}-06$ \\
\hline Subrasante & $\varepsilon \mathrm{ZZ} \rightarrow \varepsilon \mathrm{c}$ & $-264.94 \mathrm{E}-06$ & $-253.27 \mathrm{E}-06$ & $-251.67 \mathrm{E}-06$ & $-251.18 \mathrm{E}-06$ \\
\hline
\end{tabular}

Fuente: propia. 


\section{Conclusiones}

En todos los casos modelados con elementos finitos, la deformación ezz máxima en la subrasante es mayor bajo el centro de la carga dual y no bajo una llanta como lo sugiere el modelo multicapa elástico, probablemente por la carga dual y/o la misma rigidez de la estructura y de la subrasante. Las causas serán estudiadas en futuros análisis.

Si se observa con cuidado los porcentajes de variación reportados en las tablas, es posible concluir que los diseños realizados con métodos analíticos están siendo ligeramente sobredimensionados. Por ejemplo, las deformaciones åxx obtenidas del análisis con presión constante circular y presión media onda sinusoidal en la capa GB son: $84.26 \mathrm{E}-06$ y 78.61E-06 respectivamente, que en términos de número de ejes simples equivalentes con carga dual de 13T corresponde a 3620783 y $5122958 \mathrm{NE}$ con espesores de $0.180 \mathrm{~m}$ y $0.195 \mathrm{~m}$, revelando una diferencia de espesor de $0.015 \mathrm{~m}$ que constructivamente se traduce en $2 \mathrm{~cm}$ adicionales de espesor en el diseño y en consecuencia mayores costos de obra.

El método de los elementos finitos ha demostrado ser muy eficaz y en el caso particular, permitió evaluar la influencia de la presión de contacto de una carga dual sobre los parámetros de diseño de un pavimento, por lo tanto marca un camino a seguir para estudiar la sensibilidad de distintos factores que pueden afectar el diseño de pavimentos asfalticos.

Generado el interés de profundizar en la influencia de la presión de contacto de una carga dual sobre los parámetros de diseño de pavimentos asfalticos, en próximos estudios se analizarán condiciones dinámicas introduciendo resultados obtenidos de pruebas de campo a escala real.

\section{Referencias bibliográficas}

AASHTO, 2008. Mechanistic-Empirical Pavement Design Guide, A Manual of Practice. American Association of State Highway and Transportation Officials, USA.

Bonaquist, R. and Christensen, D.W., 2005. Practical procedure for developing dynamic modulus mas- ter curves for pavement structural design. Transportation Research Record, pp. 208-217.

Brown, E.R., Kandhal, P.S., Zhang, J., 2001. Performance Testing for Hot Mix Asphalt. NCAT Report No. 01-05. University of Auburn, National Center for Asphalt Technology. Auburn Alabama USA.

Davids, W. G, 2009. EverStressFE1.0 Software for 3D Finite-Element Analysis of Flexible Pavement Structures. The Washington State Department of Transportation. Washington USA.

Davids, W. G, 2009. EverStressFE1.0 Theory Manual. The Washington State Department of Transportation. Washington USA.

Davids, W. G, 2009. EverStressFE1.0 Software for 3D Finite-Element Analysis of Flexible Pavement Structures: Summary of Features and Capabilities. University of Maine, AEWC Advanced Structures and Composites Center. Maine USA.

Kringos, N., Birgisson, B., Frost, D., Wang, L., 2013. Multi-Scale Modeling and Characterization of Infrastructure Materials, Proceedings of the International RILEM Symposium: Estocolmo, Springer.

LCPC, 1994. Conception et dimensionnement des structures de chaussée, Guide technique. LCPC and SETRA, Paris.

Papagiannakis, A.T., Masad, E.A., 2008. Pavement Design and Materials. John Wiley \& Sons. Texas USA.

Rabaiottia, C., Puzrinb, A., Caprezb, M., Ozanc, C., 2013. Pavement structural heal th evaluation based on inverse analysis of three dimensional deflection bowl. International Journal of Pavement Engineering, 14(4), pp. 374-387.

Rodríguez, W., Pallares M., 2014. Modelado tridimensional de un pavimento bajo carga dual con elementos finitos. DYNA 82 (189), pp. 30-38.

Rodríguez, W. y Pallares M., 2005. Desarrollo de un modelo de elementos finitos para el diseño racional de pavimentos. Tecnura, Tecnologia y Cultura Afirmando el Conocimiento, pp. 25-37. 
Witczak, M.W., Kaloush, K., Pellinen, T., El-Basyouny, M., Von Quintus, H., 2002. NCHRPReport 465, Simple Performance Test for Superpave Mix Design. Transportation Research Board, National Research Council. Washington USA.
Xia, K., 2010. Finite element modeling of dynamic tire/ pavement interaction. Pavements and Materials: Testing and Modeling in Multiple Length Scales, pp. 204-214. 